\title{
Aplicación de las TIC en estudiantes de Ciencias de la Actividad Física y del Deporte: plataforma virtual WebCT
}

\author{
Alfonso CASTILlo-RodrígueZ \\ Universidad Pablo de Olavide de Sevilla (España) \\ acasrod1@upo.es \\ Leandro Álvarez-KUROGI \\ Universidad Internacional de La Rioja (España) \\ leandro.alvarez@unir.net
}

\begin{abstract}
Resumen
Los sistemas de enseñanza han sido modificados según la evolución de la sociedad moderna. En este estudio, se ha empleado durante 2 cursos académicos una modalidad de enseñanza mixta (presencial y virtual) con alumnos de la asignatura Estructura y Organización de Instituciones Deportivas, perteneciente a la licenciatura en Ciencias del Deporte, recurriéndose para el aprendizaje virtual a la plataforma WebCT. Los resultados indican que los alumnos han tenido apenas un $5 \%$ de incidencias con este nuevo modelo de enseñanza. Como conclusión, este modelo de enseñanza es beneficioso para el alumnado.
\end{abstract}

Palabras clave: Enseñanza-aprendizaje virtual; WebCT; plataformas virtuales pedagógicas; Ciencias del Deporte

\section{ICT application in students from Physical Activity and Sports Science: WebCT virtual platform}

\begin{abstract}
Educational systems have been modified according to the evolution of modern society. In this study, blended learning (combining face-to-face and online learning) has been used for 2 academic years with students of the subject Structure and Organization of Sports Institutions, belonging to the degree of Sports Science, by means of the virtual platform called WebCT. The results indicate that students have had only $5 \%$ of incidents with this new teaching model, which leads us to conclude that this model of education is beneficial for students.
\end{abstract}

Key Words: Virtual teaching-learning; WebCT; pedagogical virtual platforms; Sport Sciences.

\section{Referencia normalizada:}

Castillo Rodríguez, A. y Álvarez Kurogi, L. (2013) Aplicación de las TIC en estudiantes de Ciencias de la Actividad Física y del Deporte: plataforma virtual WebCT. Historia y Comunicación Social. Vol. 18. № Especial Diciembre. Págs. 279-290.

Sumario: 1. Introducción. 2. Blended Learning: aplicación en la enseñanza universitaria. 2.1. Plataformas Virtuales. 2.2. Herramienta Virtual como recurso a la enseñanza presencial. 3. Metodología semipresencial en Ciencias del Deporte. 3.1. Herramientas disponibles en la Plataforma WebCT. 4. Conclusiones. 5. Referencias bibliográficas. 


\section{Introducción}

La función pedagógica ha sido investigada por diversos científicos desde diferentes ámbitos, siendo uno de ellos es el proceso de enseñanza-aprendizaje a distancia que no requiere la asistencia física del estudiante. Ello ha emergido con la evolución tecnológica que, en consonancia con Romero (2006), tras la introducción de las Tecnologías de la Información y de la Comunicación (en adelante TIC) ha representado una notable transformación tanto en la metodología como en todos los miembros de la comunicad educativa. En consecuencia, González (2006) subraya la existencia de diversas ventajas que proporcionan las TIC, como la flexibilidad de tiempo y de entorno con acceso a internet. De la misma manera, de acuerdo con el National Center for Education Statistics (NCES, 1999), se ofertan en Estados Unidos numerosos cursos de especialización a distancia mediante instituciones tanto privadas como públicas.

En este sentido, el acceso al espacio virtual inherente al proceso pedagógico se lleva a cabo de un modo rápido, simple y eficiente. Prueba de ello es que los miembros de la comunidad educativa poseen la misma opinión en lo relativo a la utilidad de la educación virtual, considerando provechosa no sólo para la adquisición de nuevos conocimientos, sino también para el intercambio de informaciones y seguimiento de unos contenidos específicos de una asignatura en concreto, Grados, Posgrados, Cursos de formación y etc. Por ende y acorde con la sociedad actual, beneficia el perfeccionamiento y afianzamiento de algunas competencias imprescindibles, como la competencia digital; asimismo, Camerino, Coiduras y Prat (2013) lo contextualizan al considerar que nos encontramos en la sociedad de la información

\section{Blended learning: aplicación en la enseñanza universitaria}

En consonancia con González (2006), el Blended Learning -aprendizaje mezcladoasocia todo lo positivo de la enseñanza presencial con las peculiaridades del e-learning con objeto de reforzar los aspectos positivos de ambas modalidades y, consecuentemente, reducir sus puntos lánguidos; conviene destacar que e-learning hace referencia al empleo de las TIC con finalidad educativa, según Aznar, Cáceres e Hinojo (2009). En este sentido, Bartolomé Pina (2004) asevera que esta nueva concepción nació a principios de la primera década del siglo XXI, dotando al proceso pedagógico convencional una inédita perspectiva, dado que como señala Rosas (2005), se sustituyen algunas tareas por otras relacionadas con las TIC.

La finalidad de esta tipología de aprendizaje fue enmendar los inconvenientes económicos pertenecientes a la enseñanza tradicional, por lo que tal y como resalta Pascual (2003), aparte de englobar algunos aspectos concernientes a la formación tradicional, incluye chats, clases presenciales, videoconferencias y tutelas individualizadas. Camerino, Coiduras y Prat (2013) describen una serie de ventajas para el profesor que proporciona el uso de las TIC, las cuales viabilizan la aplicación del 
Blended Learning de una manera más eficaz: flexibilidad, posibilidad de interacción, rapidez, ajuste a las particularidades de los estudiantes y interdisciplinariedad; de este modo, Boneu (2007) indica que el e-learning es la última etapa de la enseñanza a distancia.

Esta conjunción de enseñanza virtual y presencial, como especifican March, Mcfadden y Price (2003) y en la misma línea que González (2006), permite afirmar que este vocablo integra los aspectos pedagógicos tradicionales y los referentes a la educación online con objeto de conferir la mejor formación, por lo que tal y como indica Vera (2008), los elementos tecnológicos constituyen la novedad de esta modalidad de enseñanza semipresencial; igualmente, el segundo autor manifiesta que su aplicación dependerá de las necesidades de cada curso, por lo que quizá esta metodología resulta eficiente fundamentalmente para aquellos estudiantes que reúnen unas cualidades que han sido bien heredadas o desarrolladas a lo largo de los años, como la motivación, organización, disciplina y lectoescritura, por lo que tal y como señala García (2005), ostenta una vertiente formativa y tecnológica.

No obstante, Bartolomé Pino (2002) describe algunos elementos que favorecen el proceso pedagógico tradicional, como la constancia relacionada con el hecho de tener que acudir a clase, la capacidad social que se adquiere a través de la interacción física con compañeros y otros miembros de la Universidad y la motivación interpersonal que puede generarse mediante la relación con otros colegas. Por ello, González (2006) subraya la inexistencia de un modelo adecuado de aplicación del Blended learning, dado que dependerá de una serie de componentes, como los objetivos didácticos, las necesidades tecnológicas y etc., además de los contenidos que deben impartirse, la etapa educativa y los conocimientos previos de los estudiantes. Por consiguiente, se contempla el aumento de los trabajos de investigación relacionados con este modelo pedagógico en la última década, que poseen relación con las características de cada alumno en función de su ciclo educativo, tal y como indica Ruíz Bolivar (2007).

La sociedad de la información en la que nos hallamos, en la que desde etapas iniciales las personas entran en contacto con las TIC, viabiliza la aplicación de este tipo de enseñanza, pudiendo ser productiva tanto para los profesores como para los estudiantes, ya que se aprovecharía sus ventajas y se eliminarían o se reducirían sus posible obstáculos. Del mismo modo, tal y como enuncia González (2006), las nuevas tecnologías permite a todos los miembros de la comunidad educativa formarse constantemente, además de posibilitar la innovación cuando sea conveniente. Aznar, Cáceres e Hinojo (2009) describen las siguientes peculiaridades características del Blended learning, a pesar de especificar el inconveniente existente relacionado con la menor interrelación física de personas: asociación de sesiones presenciales y a distancia, flexibilidad horaria, celeridad en el acceso a la información y a la comunicación, elaboración y actualización de contenidos.

En este sentido, Blended learning ha generado una respuesta positiva, puesto que puede aunar las herramientas necesarias de las dos metodologías -tradicional y online-, permitiendo el perfeccionamiento tanto de algunas habilidades más bien 
inherentes a la formación presencial -cooperación, respeto y socialización-, como de la competencia digital referente a la capacidad de buscar, detectar, cotejar y ejecutar una información puntual, acorde con Ruíz Bolívar (2007). Con todo, como precisa el mismo autor, es imprescindible tener en cuenta los conocimientos previos de los alumnos. Así, González (2006), desde la perspectiva docente, sugiere que el profesorado tenga conocimiento acerca de las nuevas tecnologías con objeto de disponer de un abanico de posibilidades para aplicar el modelo más apropiado para la adquisición de unos determinados aprendizajes, ya que de este modo se facilitará el incremento del saber de los estudiantes universitarios.

Consecuentemente, es probable que en la etapa educativa superior, la consolidación de algunas competencias se alcancen de una manera más integral, dado que los alumnos poseen un nivel superior con respecto a los ciclos educativos inferiores, aunque hemos de resaltar la existencia de diversas herramientas tecnológicas pedagógicas adaptadas a una determinada franja de edad, cuya finalidad es facilitar la adquisición de unos aprendizajes específicos acordes con el período evolutivo del estudiante, como el Jeoquiz, estrategias para la numeración, Arquímedes I, Sumar y restar y etc. Prueba de la virtud de esta metodología semipresencial es el resultado del estudio de Aznar, Cáceres e Hinojo (2009) en el que concluyen que beneficia no sólo las perspectivas de aprendizaje, sino también el nivel de adquisición de las aptitudes esenciales para su desarrollo, como el aprender a aprender y autonomía personal.

\subsection{Plataformas virtuales}

Inicialmente, cabe enfatizar que de acuerdo con Diego, Iglesias y Molina (2006), el proceso de aprendizaje a través de estas plataformas es una tipo que experimenta una continua evolución, debido a su movilidad en lo referente a contenidos y a todos los receptores viables. Su definición radica en que dichas plataformas son un software que sirven de soporte y activan el proceso de enseñanza-aprendizaje en los organismos formativos.

Por otro lado, se hace necesario puntualizar que sus albores poseen relación con la los sistemas de gestión de contenidos, cuya traducción es content management systems -CMS-, que igualmente se consideran un software, aunque designado para la administración de una página web y limitado, dado que no incluye herramientas didácticas, como indica Boneu (2007). Asimismo, este autor sostiene que todas las plataformas virtuales han de tener cuatro características necesarias, como la flexibilidad, estandarización, interactividad y escalabilidad.

En la misma línea, Comezaña y García (2005) aseveran que en el proceso referente a la formación online existen tres roles diferenciados -el docente, el discente y el técnico especializado, por lo que puede considerarse que las Universidades españolas poseen un campus virtual, el cual está generado mediante una plataforma virtual. Existen plataformas libres, como menciona González (2006) al referirse a Moodle y Webct, que de acuerdo con Muñoz-Repiso (2007), es una de las que más se utilizan en la etapa educativa universitaria, tanto es que Román Mendoza (s.f.) asegura que 
esta plataforma es recurrida en más de 55 países; Comezaña y García (2005) afirman que es una de las plataformas más conocidas y completas, además de añadir Claroline, la cual también es gratuita como Moodle y eduStance.

Igualmente, otros autores determinan a Webct como la mejor en lo referente a la funcionalidad y a la consistencia, como indican Mondéjar, Mondéjar y Vargas (2006), prueba de ello es su traducción a 15 idiomas, así como la especificación de Comezaña y García (2005) concerniente a los 2200 entidades en más de 79 países que la usan.

Por otro lado, García (2005) y González (2006) especifican que pertenecen a las dos plataformas comerciales más conocidas conjuntamente con Blackboard, siendo utilizada en el 50\% de las universidades españolas, en tanto que las más distinguidas de manera gratuita son Moodle y Claroline, a pesar de que González (2006) también cita a Dokeos y a Manhantan. Por consiguiente, puede considerarse como una de las plataformas con mayor difusión mundial.

\subsection{Herramienta virtual como recurso a la enseñanza presencial}

En relación al objetivo de este estudio de investigación, existen diversos recursos educativos como blogs, diario deportivo y página de acciones y/o ejercicios deportivos que ayudan a los profesores de la disciplina de Educación Física a impartir sus clases (Camerino, Coiduras y Prat, 2013). Además, la llegada de las tecnologías de la comunicación ha favorecido una modificación en el rol del profesor (Salinas Ibáñez, 2004), por lo que ha pasado a ser el mediador del conocimiento, desde un mero transmisor del mismo, como tradicionalmente.

Tras la posibilidad de acceder a diversas informaciones a través de internet, se ha creado herramientas y recursos cuya utilización práctica está enfocada, tanto a los docentes, con posibilidad de emplearlas como medio para conseguir un objetivo concreto del proceso de enseñanzaaprendizaje, como al estudiante, que es el otro elemento fundamental de este proceso.

Las posibilidades conseguidas gracias a las plataformas y herramientas virtuales son diversas e independientes entre sí, y se consolidan como elementos estructurales y funcionales. Sin embargo, existe la posibilidad de poseer alguna vinculación, como por ejemplo los contenidos pertenecientes a un apartado de una determinada asignatura, y, por otra parte, la calificación final obtenida en diversas partes de la asignatura en un apartado relativo al expediente académico.

De la misma forma, otro ejemplo sería que cada alumno puede intercambiar y comunicarse con otros de la misma asignatura, con cualquier contenido o tareas de la asignatura, con diversidad de formatos compatibles para las subidas de archivos, mediante un correo electrónico. Igualmente, es sabido que actualmente se ha sustituido la pizarra tradicional por pizarras digitales y a su vez, existen programas de edición de texto que mejora la calidad del anterior recurso educativo. En estos últimos años, se están creando constantemente, recursos educativos ad hoc como programas 
y páginas web que tienen por objeto ayudar a la enseñanza con modalidad presencial y a la enseñanza on-line.

Existen por tanto, diversas herramientas enfocadas más hacia el aprendizaje como por ejemplo, foros, chat, correo electrónico y componentes de sincronización y trabajo desconectado; y otras encaminadas a la gestión y a la enseñanza como por ejemplo, videoconferencias, corrección y seguimiento de los estudiantes y administración de los estudiantes y todos los cursos impartidos (Boneu, 2007). Del mismo modo, se incluye páginas on-line que permiten la divulgación de elementos didácticos como imágenes y vídeos, creando para ello, unas bases de datos que contenga dichas información, así como publicaciones sobre los temas o tópicos correspondientes.

No obstante, se ha creado el proyecto de Campus Virtual constituido por 9 universidades públicas, las cuales son exclusivas en sus pertinentes Comunidades Autónomas (La Rioja, Islas Baleares, Cantabria, Oviedo, País Vasco, Castilla - La Mancha, Extremadura, Navarra y Zaragoza), lo que beneficia la comunicación e interrelación anteriormente comentada con la posibilidad de compartir todas las asignaturas impartidas en las universidades antes mencionadas, a las cuales el alumnado posee acceso (Mondéjar, Mondéjar y Vargas, 2006). Además, en este Campus Vitual se engloba, el proyecto Profesores invitados por red, cuya finalidad es consentir a los estudiantes ver videoconferencias de un docente o inverstigador de otra universidad para completar lo impartido por el profesor responsable de la asignatura. Este Campus Virtual fue creado a través de la plataforma WebCT posibilitando a profesorado y a alumnado una serie de recursos que permite una mejor organización de la asignatura, gracias a la optimización del ritmo y del tiempo de aprendizaje, al poder acceder más rápidamente a los contenidos sin necesidad de acudir de forma presencial a la facultad concreta de la universidad correspondiente. Con este sistema, todos los estudiantes se adaptan a sus circunstancias personales, incrementan la comunicación con el profesor y amplían la comunicación entre ellos mismos, mediante herramientas como chat, coreo electrónico y foro. En el estudio de Mondéjar, Mondéjar y Vargas (2006), los alumnos se encontraban satisfechos la adaptación de este sistema de enseñanza a sus demandas y necesidades debido a la escasez de tiempo para compaginar el estudio con su actividad laboral.

Por último, todas las herramientas virtuales son eficaces, si se ajustan a los objetivos didácticos que hemos de cumplir en cada asignatura, curso..., gracias a la existencia de otras aplicaciones que pueden servir de apoyo a la docencia presencial, desde un blog educativo, revistas electrónicas, periódicos digitales, diccionarios, pizarra digital y vídeos, hasta imágenes ilustrativas, y presentaciones a través del Power Point de Microsoft Office (Microsoft Office Corporation, United States) o redes online de colaboración entre los docentes que facilita el intercambio de ideas, propuestas, conocimientos, ..., como indica García-Valcárcel Muñoz-Repiso (2007). 


\section{Metodología semipresencial en ciencias del deporte}

Los profesores pueden ofrecer a los alumnos contenidos de tipo teóricocognitivo (temas propios, libro, capítulos de libro, artículos) y prácticomotriz (enlaces a videos, sesiones de Educación Física, ejercicios de acciones motrices divididos o estructurados en función de las habilidades motrices que se refiera), gracias a la plataforma WebCT.

Durante dos años académicos (2011-2012 y 2012-2013), se ha trabajado y completado los diferentes recursos de la plataforma WebCT. Se ha considerado esta plataforma como un recurso didáctico imprescindible para la asignatura troncal de "Estructura y Organización de Instituciones Deportivas", perteneciente al quinto curso de la Licenciatura en Ciencias del Deporte en la Universidad Pablo de Olavide de Sevilla. La organización de esta asignatura en Enseñanzas Básicas, Enseñanzas Prácticas, Actividades Dirigidas y Tutorías colectivas, fue la establecida por el proyecto de Bolonia para los estudios de Grado. Todos los elementos son susceptibles de ser evaluados, aunque el último elemento (tutorías colectivas) se utilizan para la resolución de dudas del alumnado.

La creación de diversos contenidos propios, fue desarrollada por los profesores que impartían esta asignatura, dentro de cada uno de los elementos de la plataforma WebCT que configuran las asignaturas de estos estudios. Esta creación de contenidos, se encuentra fácilmente accesible a los alumnos que se encuentran matriculados en la asignatura de dicho año académico. Por otro lado, se encuentra "blindado" para las personas ajenas a este foro universitario. Además, cada uno de estos contenidos estaba planificado por el calendario académico general de la asignatura, insertado en una carpeta denominada "información de interés". Las tareas diseñadas en las enseñanzas prácticas fueron creadas con información completa para el alumnado sobre las reglas y normas para su óptimo desarrollo. Normalmente, las actividades estaban organizadas en diferentes grupos con un número de alumnos específicos, en función de la dificultad y complejidad de la actividad, por lo que para su correcto desarrollo, los alumnos se comunicaban entre ellos y se coordinaban para completar las instrucciones de estas actividades prácticas, en aras de conseguir un trabajo lleno de creatividad, organización, orden, cooperación, resolución de problemas, entre otras habilidades. En estas actividades, se proporcionaba un correo electrónico para obtener unas respuestas a nivel individual, así como diferentes patrones y normas en cuanto a formato, párrafo, extensión,..., así como, diferentes referencias bibliográficas que podían acceder (open access) para elaborar dichas tareas.

El modelo de enseñanza utilizado para la asignatura de Estructura y Organización de Instituciones Deportivas fue evaluado, teniendo en cuenta las incidencias que pudieran ocurrir en dicha plataforma, para confirmar si este modelo puede ser útil y práctico para la sociedad específica de estos estudios. Este sistema de estudio ha sido novedoso en las Ciencias de la Actividad Física y del Deporte ya que es una disciplina meramente instrumental/procedimental, y muchos autores y profesores no creyeron en este modelo desde el principio. Gracias a este modelo, concretamente 
en nuestra asignatura, los alumnos han podido aprender cómo se crea un club deportivo, saber cómo buscar subvenciones para dicho club, encontrar tipos de contrato y grupos o categorías laborales a través del II Convenio Colectivo Estatal de Instalaciones Deportivas y Gimnasios, saber el concepto de subrogación, sus características y tipos, conocer la responsabilidad civil en el deporte y comprender la estructura básica de las instituciones deportivas como son el Comité Olímpico Español (COE), Comité Olímpico Internacional (COI), las Federaciones Deportivas (internacionales, estatales y autonómicos).

En cuanto a los resultados del análisis del número de incidencias, para determinar si este modelo de enseñanza practicado es beneficioso para el alumnado y el profesorado, se han hallado un $7 \%$ en incidencias puntuales de conexión, que fue solucionado durante las 24 horas siguientes, apenas un 5\% de los alumnos tuvieron problemas para las subidas de las tareas al profesor, que fueron solucionadas con el envío de las mismas al correo electrónico. En este sentido, en resumen, durante dos años académicos, el nivel de incidencias ha sido bajo - escaso. Cada año académico se contaba con 160 alumnos/as para la realización de esta evaluación. Por tanto, la evaluación de la plataforma WebCT como recurso didáctico para la asignatura de Estructura y Organización de Instituciones Deportivas ha sido muy positiva, recomendando su uso como apoyo tanto al docente como al discente en su proceso de enseñanza-aprendizaje.

\subsection{Herramientas disponibles en la plataforma WebCT}

En la plataforma virtual WebCT existen multitud de aplicaciones, en las que en ocasiones, resulta complicado utilizarlas todas. Debido en ocasiones al desconocimiento sobre la aplicación práctica de estas aplicaciones, es, por tanto, necesario explicar las mismas.

Acerca de las herramientas de contenido, se han encontrado diversas:

a)Programa. Se ha utilizado en nuestra asignatura en los estudios de Ciencias de la Actividad Física y del Deporte. Es una aplicación en el que se puede acceder al programa real de la asignatura.

b) Glosario. Dicha aplicación es empleada para la recogida de términos claves de cada tema en la asignatura correspondiente. De esta forma, el alumno tiene al alcance una opción conceptual diferente.

c) Contenidos del curso. En ella, se condensa el grueso del temario de la asignatura. Es la aplicación más utilizada por los profesores.

d) $C D-R O M$. El profesor puede complementar algunas ideas o temas con la incorporación de un CD-ROM que comparte con sus alumnos.

En lo referente a las herramientas de comunicación, se pueden apreciar además de las aplicadas en nuestra asignatura (correo electrónico y calendario académico): 
a) Foros de debate. El profesor crea un foro a través de una pregunta relacionada con la temática deseada y realiza una primera aportación explicando al alumnado las bases de dicho foro.

b)Chat. Lo que puede asemejarse a tutorías on-line. Esta herramienta es factible para aquellos alumnos que no pueden desplazarse a las tutorías establecidas por el profesor en el campus universitario.

c) Pizarra electrónica. Este tipo de aplicaciones puede servir para la incorporación de una estrategia o táctica en nuestro campo de estudio. En otros casos, como la Cinemática,..., puede ayudar a solucionar de forma rápida un problema matemático.

En tercer lugar, se encuentran las herramientas para la evaluación. En estos dos años lectivos, se ha utilizado constantemente la herramienta de "tareas", sin embargo existen otras como:

a) Exámenes. Es una herramienta escasamente utilizada, puesto que no garantiza la capacidad individual del alumno. Sin embargo, se puede utilizar como test de control para que el alumno valore sus conocimientos de forma objetiva.

b) Autoevaluación. Es una prueba sobre la percepción del alumnado para mostrar su grado de implicación en la asignatura.

Finalmente, existen herramientas para los estudiantes como:

a) Página del estudiante. Cada alumno puede crear su propia página con sus características, intereses, necesidades,...

b) Presentaciones y trabajos en grupo. Los alumnos pueden subir sus trabajos para que otros compañeros puedan acceder a los mismos.

c) Mis calificaciones. Es la aplicación que muestra todas las calificaciones de cada alumno en función de la configuración que el profesor haya estimado apropiada para que se apruebe cada parte de la evaluación (enseñanzas básicas, enseñanzas prácticas y actividades dirigidas).

\section{Conclusiones}

El Espacio Europeo de Educación Superior (EEES) establece el modelo mixto (presencial y virtual) del proceso de enseñanza-aprendizaje como fundamental para las enseñanzas universitarias, gracias a la expansión de las Nuevas Tecnologías. En el presente estudio se ha analizado si este modelo mixto permite ajustarse a las necesidades actuales del alumnado de Ciencias de la Actividad Física y del Deporte, ya que tradicionalmente, esta disciplina está relacionada con el aprendizaje procedimental en un contexto o medio físico determinado. Sin embargo, existen asignaturas en la que los alumnos pueden examinar, analizar y desarrollar sus capacidades a través de unos contenidos que se muestran en la plataforma virtual. Estos contenidos pueden 
ser de ámbito conceptual, aunque también procedimental y actitudinal, para la formación integral del futuro Licenciado en Educación Física y mostrar una organización y planificación de los recursos didácticos necesarios.

Se ha demostrado, en primer lugar, que siguiendo este modelo de enseñanza tiene como consecuencia un ahorro económico a los alumnos, debido a la ausencia de fotocopias de los contenidos y actividades de la asignatura, si bien es cierto, que también permite colaborar con el proceso ecológico y ahorro de papel, muy necesario en la actualidad. De esta forma, estos contenidos y tareas se encuentran subidos a la plataforma durante todo el año académico.

En cuanto al ámbito actitudinal, los alumnos que practican este modelo de enseñanza-aprendizaje, aprenden a ser más responsables, valor que está relacionado con el futuro ámbito laboral de los alumnos, ya que solicitan la competencia de autonomía e iniciativa personal. Desde el principio del curso, los alumnos pueden saber qué días son presenciales y cuáles no. Pueden ver dicha información las veces necesarias y encontrar la planificación en cuanto a enseñanzas prácticas y actividades dirigidas.

Como conclusión de este estudio, se reconoce la plataforma educativa WebCT, como recurso ideal para los alumnos universitarios de la actualidad. Se ha evaluado un modelo de enseñanza-aprendizaje mixto, como se exige en los nuevos estudios de Grado, utilizando esta plataforma como soporte fundamental de la asignatura de la antigua Licenciatura, durante dos años académicos. Esta plataforma ha sido muy estable, con un nivel de incidencias muy ínfimo, permitiendo la consecución de los objetivos establecidos en la asignatura específica, además, de conseguir nuevos valores relacionados con el ámbito laboral, sea en el ámbito educativo, de rendimiento o de gestión deportiva.

\section{Referencias bibliográficas}

BARTOLOMÉ PINA, A. (2002). Universidades en la Red. ¿Universidad presencial o virtual?, en Revista Crítica, LII896, nº 896. Disponible en: www.lmi.ub.es/personal/bartolome/articuloshtml/bartolomeSPcritica02.pdf. Consultado el [25-032013].

BARTOLOMÉ PINA, A. (2004). Blended learning. Conceptos básicos, en Píxel-bit. Revista de medios y educación, $\mathrm{n}^{\circ}$ 23. Disponible en: www.sav.us.es/pixelbit/ pixelbit/articulos/n23/n23art/art2301.htm. [19-05-2013].

BONEU, J. M. (2007). Plataformas abiertas de e-learning para el soporte de contenidos educativos abiertos, en Revista de universidad y sociedad del conocimiento, $\mathrm{n}^{\mathrm{o}}$ 4. Disponible en: www.raco.cat/index.php/RUSC/article/view/58133/68225. [05-03-2012].

CAMERINO FOGUET, O.; COIDURAS RODRÍGUEZ, J. L.; PRAT AMBRÓS, Q. (2013). Introducción de las TIC en educación física. Estudio descriptivo sobre la 
situación actual, en Apunts. Educación Física y Deportes, n 113 . Disponible en: http://www.observesport.com/desktop/images/docu/3walelni.pdf [01-10-2013].

GARCÍA PEÑALVO, F. J. (2005). Estado actual de los sistemas e-learning, en Teoría de la Educación: Educación y Cultura en la Sociedad de la Información, n ${ }^{\circ} 6$. Disponible en: http://campus.usal.es/ teoriaeducacion/rev_numero_06_2/n6_02_ art garcia penalvo.htm. [08-04-2013].

GONZẢLEZ MARIÑO, J. C. (2006). B-Learning utilizando software libre, una alternativa viable en Educación Superior, en Revista Complutense de Educación, $\mathrm{n}^{\mathrm{o}}$ 1. Disponible en: http://revistas.ucm.es/index.php/RCED/article/view/ RCED0606120121A [19-05-2013].

GARCÍA-VALCÁRCEL MUÑOZ-REPISO, A. (2007). Herramientas tecnológicas para mejorar la docencia universitaria. Una reflexión desde la experiencia y la investigación, en Revista iberoamericana de educación a distancia, $\mathrm{n}^{\circ} 2$. Disponible en: http://e-spacio.uned.es/fez/eserv.php?pid=bibliuned:20548\&dsID=herramientas_tecnologicas.pdf. [15-11-2012].

MONDÉJAR JIMÉNEZ, J.; MONDÉJAR JIMÉNEZ, J. A.; VARGAS VARGAS, M. (2006). Implantación de la metodología e-learning en la docencia universitaria: una experiencia a través del proyecto Campus Virtual, en Revista Latinoamericana de Tecnología Educativa, no 1. Disponible en: http://dialnet.unirioja.es/ servlet/articulo?codigo=2229170. [03-11-2012].

MARCH, G. E.; MCFADDEN, A. C.; PRICE, B. J. (2003). Blended Instruction: Adapting Conventional Instruction for Large Classes, en Online Journal of Distance Learning Administration, $\mathrm{n}^{\mathrm{o}}$ IV. Disponible en: http://www.westga.edu/ distance/ojdla/winter64/marsh64.htm. [12-12-2012].

NATIONAL CENTER FOR EDUCATION STATISTICS (1999). Digest of Education Statistics. Disponible en http://nces.ed.gov/pubs2000/digest99/. [02-09-2013].

PASCUAL, M. P. (2003). El Blended learning reduce el ahorro de la formación on-line pero gana en calidad, en Educaweb, $\mathrm{n}^{\circ}$ 69. Disponible en: http://www. educaweb.com/esp/servicios/monografico/formacionvirtual/1181108.asp [07-102012].

ROMÁN-MENDOZA, E. (s.f.). El desarrollo de cursos a distancia en la World Wide Web mediante plataformas virtuales: "WebCT" en el mundo universitario norteamericano. Disponible en: http://cvc.cervantes.es/ensenanza/formacion_virtual/ metodologia/roman.htm. [07-02-2011].

RUÍZ BOLÍVAR, C. (2007). El blended learning como evaluación de una experiencia de aprendizaje en el nivel de posgrado, en Teoría de la Educación: Educación y Cultura en la Sociedad de la Información, nº 8. Disponible en: http://campus.usal. es/ teoriaeducacion/rev_numero_08_03/n8_03_ruiz_bolivar.pdf. [28-06-2013].

SALINAS IBÁÑEZ, J. (200̄4). Innovación docente y uso de las TIC en la enseñanza universitaria, en Revista de universidad y sociedad del conocimiento, $\mathrm{n}^{\mathrm{o}} 1$. Disponible en: http://www.uoc.edu/rusc/dt/esp/salinas1104.pdf. [29-10-2012].

VERA, F. (2008). La modalidad blended-learning en la educación superior. Disponible en: http://www.utemvirtual.cl/nodoeducativo/wp-content/uploads/2009/03/ fvera_2.pdf. [16-10-2013]. 


\section{Los autores}

Alfonso Castillo-Rodríguez. Profesor Asociado en la Universidad Pablo de Olavide de Sevilla y en la Universidad Internacional de la Rioja. Asignaturas impartidas: Estructura y Organización de las Instituciones Deportivas, Deporte y Recreación, Entrenamiento Deportivo, Didáctica de la Actividad Física y del Deporte, Juegos Motores y Prácticum. Líneas de investigación: Fisiología y Psicología del deporte, Condición física en adolescentes, adultos y mayores, Didáctica de la Actividad Física y del Deporte. Diversas publicaciones en libros, capítulos de libro, revistas, congresos, ...

Leandro Álvarez Kurogi. Defensor del Estudiante en la Universidad Internacional de la Rioja. Profesor en titulaciones de postgrados en Universidades Públicas. Líneas de investigación: Análisis del rendimiento en deportes colectivos, Condición física en adolescentes, adultos y mayores, Didáctica de la Actividad Física y del Deporte. Publicaciones de prestigio internacional (JCR). 